\title{
Prevalence of Antimicrobial Sensitivity and resistant Pattern of Gram Positive Cluster Forming Cocci in Clinical Samples
}

\author{
Zinnat Shahina ${ }^{1}$, A. H. M. Ishaque Chowdhury ${ }^{1}$, Md. Arifuzzaman ${ }^{2} *$ \\ ${ }^{1}$ Department of Microbiology, University of Science \& Technology Chittagong (USTC),Chittagong, Bangladesh \\ ${ }^{2}$ Department of Biochemistry \& Biotechnology, University of Science \& Technology Chittagong (USTC), \\ Chittagong, Bangladesh
}

\begin{abstract}
Purpose: Drug resistance is a burning issue in bacterial kingdom and in appropriate use of antibiotics is one of the most important factors that could affect the increasing patterns of resistance. The aim of the present study is to ascertain the current situation of growing antibiotic resistance against bacteria, ex-gram positive cluster forming cocci. Materials and Methods: Various clinical samples such as pus, sputum, throat swab, high vaginal fluid, prosthetic swab, wound swab, ear swab were taken from776 subjects and cultured on nutrient agar and Mac Conkey's agar. After morphological identification 271 isolates were tested for antimicrobial susceptibility testing. Results: In this study, Antibiotic susceptibility testing was done by using 271 patients sample out of 363 positive growth culture which was collected from pus. It stated that male and female ratio for infection was 1:0.6 and resistance are high in the age group 21-40 years. Observation of susceptibility test indicate that ampicillin, amoxycillin and co-trimoxazole showed increase rate of resistance which were 28 , 21 and $15 \%$ respectively. On the other hand, cefuroxime and ceftriaxone showed significantly sensitive which belongs to cephalosporin second and third generation group. Conclusion: It is very important to reduce frequent misuse; inadequate dosages and easy availability of antimicrobials to keep away human generation from emerge of antibiotic resistance.
\end{abstract}

Key words: Antibiotic susceptibility, Gram positive cluster forming cocci, Gram stain, Pus.

\section{Introduction}

Staphylococcus and Streptococcus are the two medically important gram positive organism but they are distinguished by two main criteria. Microscopic observation shows Staphylococci appear in grapelike clusters, whereas Streptococci are in chains. On the other hand in biochemical nature Staphylococci produce catalase (i.e., they degrade hydrogen peroxide), whereas Streptococci do not. Among the staphylococci species three are of human pathogens: S. aureus, S. epidermidis, and S. saprophyticus [1].

S. aureus produces a carotenoid pigment that imparts a golden color to its colonies. This pigment enhances the pathogenicity of the organism by inactivating the microbicidal effect of superoxides and other reactive oxygen species within neutrophils. S. epidermidis does not synthesize this pigment and produces white colonies. The virulence of $S$. epidermidis is significantly less than that of $S$. aureus which makes it more pathogenic than other two[1].

Humans are the reservoir for Staphylococci. The nose is the main site of colonization of S. aureus and approximately $30 \%$ of people are colonized at any one time. Chronic nasal carriage increases the risk of infection by S. aureus. The skin, especially of hospital personnel and patients, is also a common site of S. aureus colonization. Hand contact is an important mode of transmission and hand washing decreases transmission. $S$. aureus is also found in the vagina of approximately 5\% of women, which predisposes them to toxic shock syndrome. Additional sources of staphylococcal infection are shedding from human lesions and fomites such as towels and clothing contaminated by these lesions [1].

Staphylococcus aureus causes abscesses, various pyogenic infections (e.g., endocarditis, septic arthritis, and osteomyelitis), food poisoning, scalded skin syndrome, and toxic shock syndrome. It is one of the most common causes of hospital-acquired pneumonia, septicemia, and surgical-wound infections. It is an important cause of skin infections, such as folliculitis, cellulitis, and impetigo [1]. The problem with Staphylococcus aureus became more complicated when it was found that it quickly developed resistance and was capable of producing many antibiotic resistant strains. This is very common in hospitals where drug resistant "hospital strains" have caused S. aureus infection outbreaks resulting in deaths in surgical units and newborn nurseries [2].Antibiotic resistance leads to prolonged hospital stay that increased morbidity and mortality rate.

The emergence of antibiotic resistance poses an urgent medical problem worldwide. Current antibiotics target a small set of proteins essential for bacterial survival. As a result, antibiotic resistant strains are subjected to a strong positive selection pressure[3]. Inappropriate and excessive use of antibiotics have contributed to the emergence of pathogens that are highly resistant to most currently available antibiotics [4][5][6]. The novel approach of inhibiting pathogen virulence while minimizing the selection pressure for resistance holds great 
promise as an alternative to traditional antibiotic treatment [3]. Knowledge of the pattern of antibiotic resistance among isolates is very important both clinically and epidemiologically. The results of antimicrobial resistance patterns are of great concern due to these predominant bacterial isolates which are highly resistant to commonly available antimicrobial agents [7].

The study was conducted to determine the prevalence and antibiotic susceptibility pattern of gram positive cluster forming cocci which was supposed to be related with Staphylococcus aureus as their morphological character.

\section{Material and Methods}

This study based on retrospective data of sample sent from different wards and OPDs of Bango Bandhu Memorial Hospital (BBMH), Chittagong, Bangladesh. A total number of 363 different types of Microorganisms were isolated from 776. Rest of 413 specimens showed no growth. Various clinical samples such as pus, sputum, throat swab, high vaginal fluid, prosthetic swab, wound swab, ear swab were used. The specimen were collected from 2007-2011.

In microbiology laboratory Swab were cultured on nutrient agar and Mac Conkey's agar for 24-48 hours. Observation of gram staining showed different morphology such as gram positive cluster forming cocci, gram positive chain forming cocci and gram negative single or chain forming rods.

Antimicrobial susceptibility testing was performed on Muller-Hinton(MH) agar by disc-diffusion method against the following antibiotics- ampicillin, amoxicillin, doxycycline, cefradine, cephalexin, cotrimoxazole, cefuroxime, cefixime, ceftriaxone and ciprofloxacin according to clinical and laboratory standard Institute[8] guide lines only on those isolates which morphologically showed gram positive cluster forming cocci.

\section{Results}

Out of 776 clinical specimen, 363 were found positive bacterial growth, rest of the 413 showed no growth on nutrient agar media. After gram staining of 363 microbial isolates, 271 showed cluster forming gram positive cocci, 28 showed chain forming gram positive cocci and 64 showed gram negative rods including short, long, single or chain(Table:1). The frequency of gram positive and gram negative bacteria were $75 \%$ and $18 \%$ respectively. Isolation of gram positive clusters were maximum (213) in pus sample (Table: 1).

Table 1: Frequency of various isolates in various specimens.

\begin{tabular}{|c|c|c|c|c|c|c|c|c|}
\hline \multirow{2}{*}{$\begin{array}{l}\text { Morphology of } \\
\text { the isolates }\end{array}$} & \multicolumn{8}{|c|}{ Name of the specimens } \\
\hline & Pus & Sputum & $\begin{array}{l}\text { Throat } \\
\text { swab }\end{array}$ & $\begin{array}{l}\text { High } \\
\text { vaginal } \\
\text { fluid }\end{array}$ & $\begin{array}{l}\text { Prosthetic } \\
\text { swab }\end{array}$ & $\begin{array}{l}\text { Wound } \\
\text { swab }\end{array}$ & $\begin{array}{l}\text { Ear } \\
\text { swab }\end{array}$ & Total \\
\hline $\begin{array}{l}\text { Gram positive } \\
\text { cluster forming } \\
\text { cocci }\end{array}$ & 213 & 35 & 10 & 5 & 2 & 5 & 1 & 271 \\
\hline $\begin{array}{l}\text { Gram positive } \\
\text { chain forming } \\
\text { cocci }\end{array}$ & 8 & 11 & 9 & 0 & 0 & 1 & 0 & 28 \\
\hline $\begin{array}{l}\text { Gram negative } \\
\text { rods }\end{array}$ & 45 & & 0 & 0 & 12 & 5 & 2 & 64 \\
\hline Total & \multicolumn{8}{|c|}{363} \\
\hline
\end{tabular}

Data was categorized according to the parameters namely sex, age and type of specimen. Among 271 patients who were infected to gram positive cluster forming cocci it was observed $169(62.4 \%)$ in males and $102(37.6 \%)$ in female patients, male to female ratio 1:0.6 (Figure: 1).

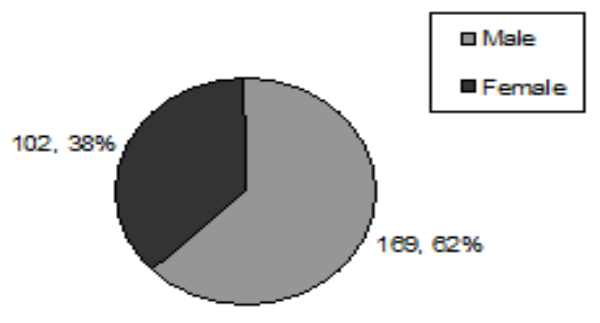

Figure 1: Frequency of Gram positive cluster forming cocci in gender groups, $\mathbf{n = 2 7 1}$. 
The sensitivity test was carried out by using isolates of gram positive cluster forming cocci with 10 antibiotics such as ampicillin, amoxicillin, doxycycline, cefradine,cephalexin,co-trimoxazole,cefuroxime, cefixime, ceftriaxone and ciprofloxacin by disc diffusion technique. Cefuroxime and ceftriaxone showed highest sensitivity. Here the level of sensitivity for low, moderate and highest growth is $50 \%$. Observation of these 3 levels showed that ciprofloxacin took second place followed by doxycycline (Figure: 2)

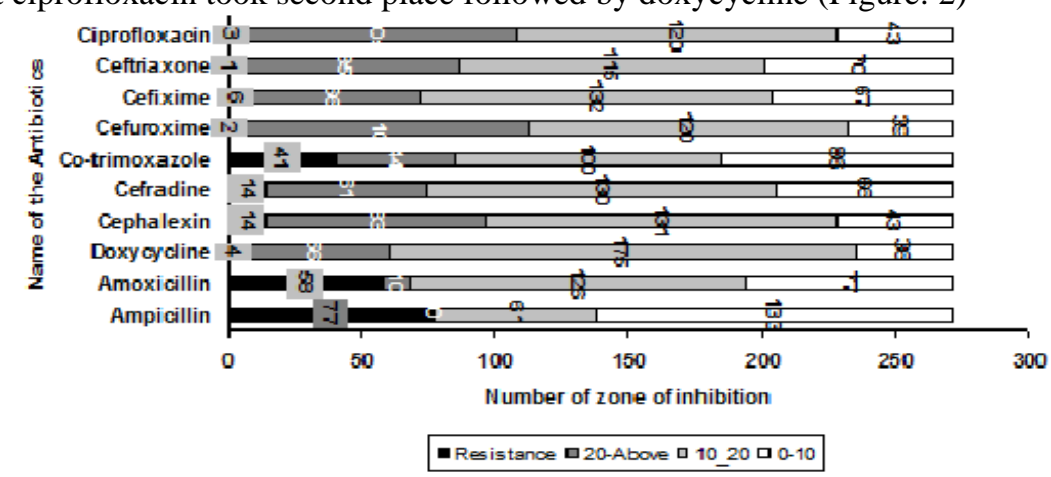

Figure 2: Antibiotic sensitivity to the selected isolates from pus sample in this study. The sensitivity of all antibiotics showed by bar columns.0-10,10-20 and above $20 \mathrm{~mm}$ represents the zone of inhibition by the antibiotics on the culture plates.

Furthermore, we have analyzed the antibiotic resistant profile of 271 isolates (Figure 3). It observed that, among study populations ampicillin, amoxicillin, cefradine, cephalexin, co-trimoxazole and cefixime showed resistance. The resistance pattern of the antibiotics was comparably high of ampicillin, amoxycillin and co-trimoxazole which were 34, 26 and 19\% respectively. The resistance pattern of other antibiotics such as cephalexin, cefradine, cefixime and doxycycline were comparably low which were 7,6, 3 and $2 \%$ respectively. The antibiotic resistant data also showed that among the cephalosporin groups second generation group cefuroxime and third generation ceftriaxone showed highest sensitivity as they showed lower resistance than others. After that third generation cefixime showed second most highest sensitivity followed by the first generation group cephradine and cephalexin respectively (Figure:3).

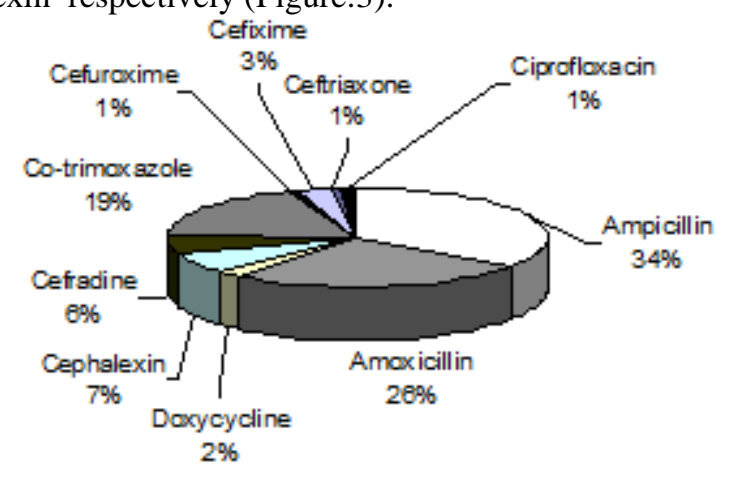

\section{Figure 3: Percentage of antibiotic resistance for gram positive cluster forming cocci in this} study.

To analyze the prevalence of resistance of infections at different age group, the present data stated that, the onset of resistance were high at the age between $21-40$ years $(38.37 \%)$. On the other hand in case of age group 0-20,41-60 and above 60 the prevalence of resistance were 28.98,27.30 and 10.33\% respectively (Figure: 4).

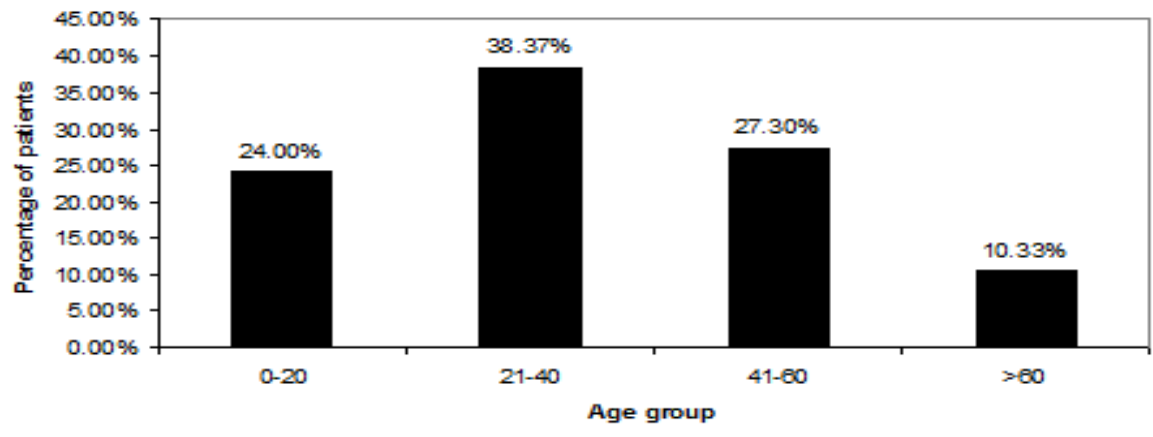




\section{Figure 4: Incidence of antibiotic resistance in various age groups.}

\section{Discussion}

In this study 776 clinical sample were analyzed where 363 showed positive bacterial growth. We conducted further study of antibiotic susceptibility and resistant profile of 271 bacterial isolates which were morphologically similar to Staphylococcus Species. As they appeared gram positive cluster form. The prevalence of gram positive cocci were higher $(75 \%)$ than the gram negative rods $(18 \%)$. These findings resembles with the findings of the researcher [9] who reported that the higher growth of gram positive bacteria(51\%) and lower growth of gram negative bacteria(49\%) among 261 positive samples. The incidence of growth was higher in pus sample (213) which were accordance with study of Qureshi[10] and Zafar [9].

Gender basis observation of 271 isolates which showed high frequency among Male patients, 62\% than Female patients, 39\% (Fig.1). Male to female ratio was 1:0.6 which is consistence with earlier work by Mahmood [11] and L D Kitara [12] but disagrees with the findings of Idighri [13], that females (32\%) were higher ratio than the male( $30 \%)$.

A total of 10 antibiotics used for antibiotic susceptibility test which revealed that the isolates showed a marked sensitivity to ceftriaxone and cefuroxime followed by ciprofloxacin and doxycycline (Fig. 2).In current studies, second generation cephalosporin group cefuroxime and third generation group ceftriaxone showed highest sensitivity than other generation of this group which is consistence with earlier report of Zafar [9] where he reported $73 \%$ sensitivity against cefotaxime and also with the results of mehmood [14], where he found $97.8 \%$ sensitivity. cefotaxime is the third generation cephalosporin group and another member of this generation which was tested by us was ceftriaxone showed $99.60 \%$ sensitivity.

In our syudy with the resistant profile of 271 clinical samples (fig.4), the data indicated that our isolates were resistant to ampicilin, amoxicilin and co-trimoxazole. This investigation accords Ramakrisnha [15] where he documented the resistance of ampicilin(95\%) and amoxicilin(75\%) for MRSA and also with the previous results of L D Kitara[12], where he showed the resistance of ampicilin was $60 \%$ and co-trimoxazole $53 \%$. Where as in our result, persistence of resistant found for ampicilin(28\%), amoxicilin(21\%) and cotrimoxazole(15\%).We also found out that the incidence of resistance was higher in age groups 21-40 years. This result agree with idighri, M.N, 2012 where he found highest percentage of isolate at the age group of 21$30(50 \%)$ and $31-40(40 \%)$. There is a probability of getting infection because of changing life style in that age groups.

This assertion can further be strengthened by the high level of antibiotic abuse in our locality, arising from self medication which are often associated with inadequate dosage and failure to comply to treatment [16] and availability of antibiotics to consumers across the counters with or without prescription [17][18].As antibiotic resistance grow day by day, it is important to use suitable antibiotic after proper laboratory diagnosis such as culture and antibiotic susceptibility tests and treatment should be depend on it.

\section{Conclusion}

The emerge of antibiotic resistance has been a problem day by day because of overusing antibiotics. It is needed to be aware of this problem to avoiding inappropriate use, frequent misuse, inadequate dosages, easy availability of antimicrobials and must be designed the treatment schedule after proper laboratory investigation.

\section{References}

[1]. Levinson W. Medical Microbiology and Immunology.Examination and Board Review.9 ${ }^{\text {th }}$ Edn., (McGraw-Hill,New York) 2006; 106-109.

[2]. Meyers HF, Ernest J, Allan G.A review of Medical Pharmacology Lange Medical Publishers. 1970; 49:467-474. 50:475-581

[3]. Waldor MK. Disarming pathogens-a new approach for antibiotic development. N. Engl. J. Med. 2006; 354: $296-297$.

[4]. Alanis AJ. Resistance to antibiotics: are we in the post-antibiotic era? Arch. Med. Res. 2005; 36: 697-705.

[5]. Bax R, Mullan N, Verhoef J. The millennium bugs-the need for and development of new antibacterials. Int. J. Antimicrob. Agents. 2000;16: 51-59.

[6]. Norrby SR, Nord CE, Finch R. Lack of development of new antimicrobial drugs: a potential serious threat to public health. Lancet Infect. Dis. 2005;5: 115-119.

[7]. Ojulong J, Mwambu TP, Jalooba M, Bwanga F, Kaddu-Mulindwa DH. Relative prevalence Methicillin-Resistant Staphylococcus aureus and its susceptibility pattern in Mulago Hospital, Kampala, Uganda. Tanzania J Health Research. 2009;11(3):149-153.

[8]. 8.Clinical and Laboratory Standard Institute,(2005).Performance Standards for Antimicrobial susceptibility testing: Fifteenth Informational supplement.Clinical and Laboratory Standards Institute, Wayne,PA.

[9]. Zafar A, Ejaz H, Ahmed JM, Javed A. Frequency and Antimicrobial Susceptibility of Staphylococcus Aureus isolated from pus Samples of Paediatric patients. Pjmhsonline 2012; vol.6(3): 32-35.

[10]. http:/pjmhonline.com/JanMarch2012/frequency_and_antimicrobial_susceptibility.httm

[11]. Qureshi AH, Rafi S, Qureshi SM, Ali AM. The current susceptibility patterns of Methicillin resistant Staphylococcus aureus to conventional anti Staphylococcus antimicrobials at Rawalpindi. Pak J Med Sci.2004; 20: 361-364.

[12]. Mahmood K, Tahir T, Jameel T, Ziauddin A and Alam H F. Incidence of Methicillin-resistant Staphylococcus aureus (MRSA) causing nosocomial infection in a tertiary care hospital.Annals.2010;vol16(2):91-96.

[13]. Kitara LD, Anywar Ad, Acullu D, Odongo-Aginya E, Aloyo J and Fendu M. Antibiotic susceptibility of Staphylococcus aureus in suppurative lesions in Lacor Hospital, Uganda. African Health sci.2011;11(S1): S34-S39. 
[14]. Idighri M N, Nedolisa A C and Egbujo E C. Antimicrobial susceptibility pattern of Staphylococcus aureus isolated from surgical wound of patients in Jos University Teaching hospital, North central Nigeria. E- international scientific research journal. 2012;IV(1): ISSN 2094-1749.

[15]. Mehmood A, Karamat A K and Butt T. Neonatal sepsis:high antibiotic resistance of the bacterial pathogens in a neonatal intensive care unit in karachi. J. Pak. Med. Assoc.2002; 8: 348-350.

[16]. Ramakrishna D.Prevalence and multi drug resistance of Staphylococcus aureus in the hospitals of Gulbarga and Raichur districts.Recent research in Science and Technology. 2012;4(2): 27-29.

[17]. Odegbemi T. The use and abuse of antibiotics. Nig. Med. Pract. 1981;1(1):5-8.

[18]. Adekeye D. Resistance of Staphylococcus aureus of man an other animal to five antibiotics commonly used in Nigeria. Nig. Med. J. 1979;9: 195-197.

[19]. Paul MO, Aderibigbe DA, Sule C Z, Lam Kanra AA. Antimicrobial sensitivity pattern of hospital and non hospital strains of Staphylococcus aureus isolated from nasal carrier. J.Hyg.1982;89:253-260. 